\title{
Role of WBC Scattergram, Histogram and Platelet Indices in Diagnosis of Malaria
}

\section{ABSTRACT}

Introduction: Malaria, a parasitic disease affecting more than 300 million people every year can sometimes be most serious and fatal too. It continues to be a major global health issue. Detection of malaria by light microscopy is the gold standard, which requires time and an expertise. In endemic areas, it is impractical to manually screen all peripheral blood films for the parasite and poses a challenge to all the laboratories, especially when the parasitic index is low. A rapid and cost effective screening of malaria is possible with the automated analysers, which provides a hint and prevents the misdiagnosis.

Aim: To study the changes in WBC Scattergram, Histogram and Platelet indices in Malaria cases and compare with normal individuals.

Materials and Methods: Prospective study was done in which blood samples were analysed using Beckman coulter LH-780. The scattergram, histogram, platelet count, mean platelet volume, platelet distribution width and Plateletcrit (PCT) of both positive and negative cases were recorded. The abnormal scattergrams and additional peaks in WBC histograms along with platelet indices were compared with peripheral smear examination. Smears were made, stained with Leishman's stain and examined. Statistical analysis was done by using software SPSS version 20. Fisher-exact test was applied to calculate the $\mathrm{p}$-value and $<0.05$ was considered as significant.

Results: Suspected malaria cases were 822, of which $74(9 \%)$ cases were positive on peripheral smear examination. Abnormal WBC scattergrams were observed in $90.5 \%$ of cases with malaria. Abnormal WBC histogram peaks were noted in $97.2 \%$ cases. Of the haematological abnormalities studied, thrombocytopenia was seen in $94.5 \%$ of cases. Mean Platelet Volume (MPV) was normal in $83.7 \%$ of cases. PCT was reduced in all cases $(100 \%)$. Platelet Distribution Width (PDW) was increased in $98.6 \%$ of cases.

Conclusion: An abnormal scattergram, abnormal histogram along with thrombocytopenia, low PCT and increased PDW can predict presence of malaria. Although automated analysers are not a screening tool for evaluation of malaria, most of the laboratories in India have cell counters but awareness is lacking regarding specific patterns of malarial infection in the histogram and scattergram.

\section{INTRODUCTION}

Malaria infecton is not an uncommon parasitic disease in humans affecting 103 countries worldwide [1]. South-East Asian countries like India, Myanmar and Indonesia account for approximately 94\% of the reported malaria cases, with India carrying a high burden of the disease, still reporting $65 \%$ of the cases [2].

Clinical diagnoses of malarial infection from other tropical infections, based on patients' signs and symptoms or physicians' findings, may be difficult [3]. Definitive diagnosis is essential for the management and control of malaria. It has been shown that traditional methods for diagnosing malaria remain problematic with many limitations [4]. The detection of malaria by light microscopy of stained smears has remained the gold standard. Many haematological abnormalities are associated with malaria, however, none of these abnormalities are specific [5].

$P$. falciparum as well as $P$. vivax is known to cause significant hematological changes with high occurrence of thrombocytopenia, anaemia and lymphopenia [6]. The automation in haematology helps in analysing various parameters which helps the pathologist to concentrate more on the peripheral blood smear to search for malarial parasite, thus allow immediate start of specific therapy of the patient $[7,8]$.

Monocytes and lymphocytes are known to get activated by malarial parasites, which are reflected as the heterogeneity of the volume in scattergrams along with infected RBC's depicted in the nonWBC's areas of the scattergram called ghost areas. Thus these changes in the volume, conductivity and scatter properties can be easily detected in automated analysers having Volume Conductivity
Scatter (VCS) technology. "Suspect malaria" flags can be seen due to abnormal WBC histogram peaks. A flag would be a useful indicator to suspect the presence of malarial parasites which can be used as a routine parameter in the diagnosis of malaria [9].

Platelet indices are altered during acute and symptomatic infection by $P$. vivax. The elevation of MPV and PDW, and reduction of PCT are related potential risk factors for evolution into severe malaria [10].

Low platelet count $\left(<150 \times 10^{9} / \mathrm{L}\right)$ is an excellent haematological parameter for presumptive diagnosis of malaria. Depolarised Laser Light (DLL) can be helpful in the diagnosis of acute malaria, but cannot replace the existing methods of diagnosis. DLL-based malaria diagnosis is particularly helpful in screening of malaria in situations like screening of blood donors. There is no significant difference in the sensitivity and the specificity of haematological parameters in patients of $P$. vivax and $P$. falciparum malaria [1]

Laboratory findings like an abnormal WBC scattergram with thrombocytopenia in a febrile patient help the pathologist to suspect the diagnosis of malaria [2]. WBC/BASO scattergram along with presence of thrombocytopenia are useful in the presumptive diagnosis of $P$. vivax [11]

Different studies have taken into different parameters to diagnose malaria infection using cell coulters. The present study was conducted to compare multiple parameters like, the WBC scattergram, histogram and platelet indices in normal individuals and those with malaria, so that it can be used as a powerful tool to suspect malaria and search for the same diligently, so that early diagnosis and treatment prevents the complications associated with malaria. 


\section{MATERIALS AND METHODS}

A prospective study was conducted over a period of 8 months from March 2016 to October 2016 after obtaining the ethical clearance from the institute.

Venous blood samples from 822 patients of all age groups with clinical suspicion of malaria during the study period, who presented with fever and chills, were collected in EDTA vaccutainers with the patients' consent. Cell counts were done using the Beckman LH 780 (Coulter) haematology analyser. Complete blood counts along with WBC scattergram, WBC histogram, platelet count, MPV, PDW and PCT of both positive and negative cases were critically analysed. Smears were made and stained with Leishman's stain. Malarial parasites were detected and species identified.

\section{STATISTICAL ANALYSIS}

Statistical analysis was done by using software SPSS version 20. Fisher exact test was applied to calculate the $p$-value and $<0.05$ was considered as significant.

\section{RESULTS}

Suspected malaria cases with acute febrile illness were 822, of which $74(9 \%)$ cases were diagnosed positive for malaria parasite on peripheral smear examination. Among the positive cases, $55.5 \%$ (41) cases were seen in males and rest $44.5 \%$ (33) cases in females. Age range was 6 to 63 years.

As depicted in [Table/Fig-1], majority of the patients had Plasmodium vivax (90.5\%). Almost all the cases showed one or more abnormalities. Abnormal WBC scattergrams [Table/Fig-2] were observed in 90.5\% of malaria cases with a p-value of 0.001 . Abnormal WBC histogram [Table/Fig-3] peaks were noted in $97.2 \%$ cases demonstrating a peak at the threshold of the histogram with a p-value of 0.001 .

\begin{tabular}{|l|c|c|}
\hline Species & Number of cases (74) & Percentage \\
\hline Plasmodium vivax & 67 & $90.5 \%$ \\
\hline Plasmodium falciparum & 05 & $6.7 \%$ \\
\hline Mixed infections & 02 & $2.7 \%$ \\
\hline
\end{tabular}
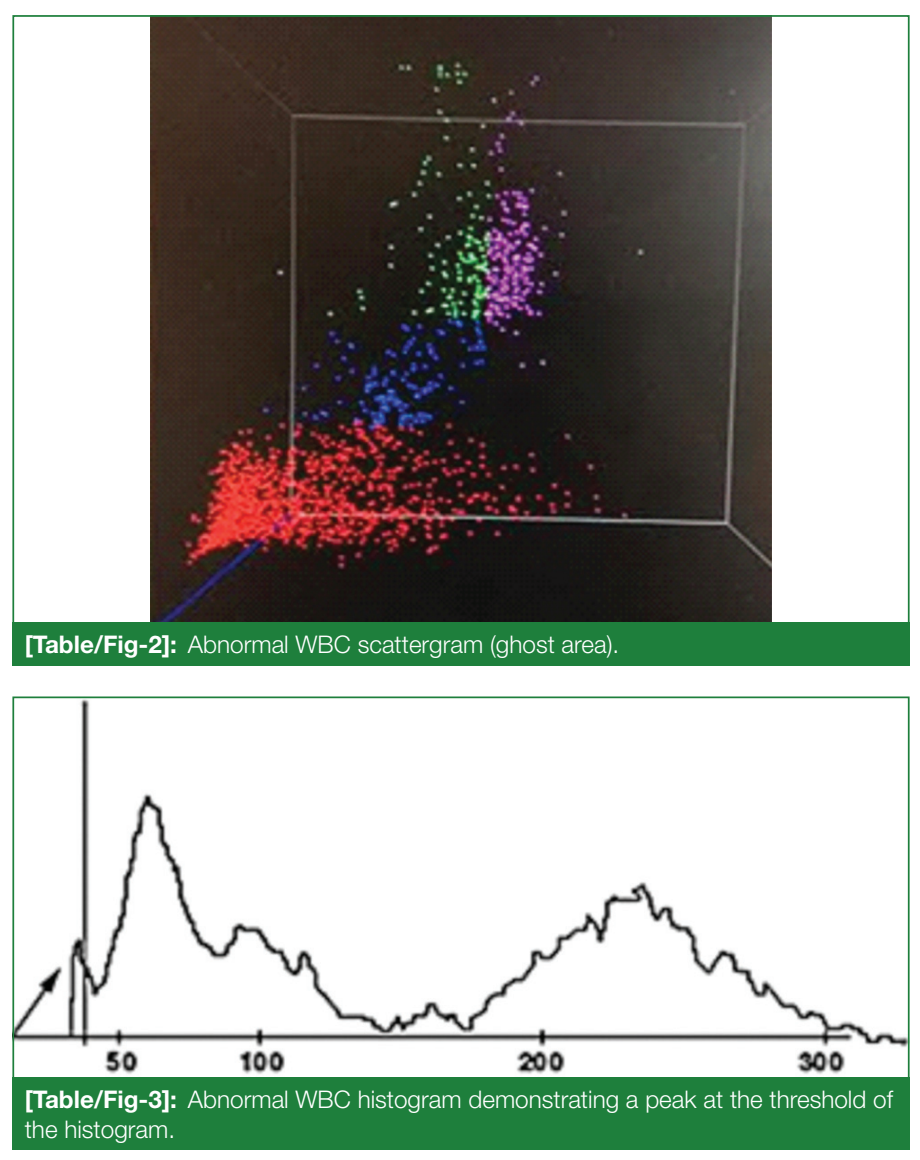

Of the haematological abnormalities studied, thrombocytopenia was seen in $94.5 \%$ of cases with a p-value $<0.001$. MPV was normal in $83.7 \%$ of cases with $p$-value of 0.106 . PCT was reduced all cases (100\%) with $p$-value $<0.001$. PDW was increased in $98.6 \%$ of cases with $p$-value of 0.004 .

\section{DISCUSSION}

Malaria pathogenesis leading to anemia is complex, multi factorial as well incompletely understood. It is believed to result from a combination of haemolysis of parasitised red blood cells; accelerated removal of both parasitised and innocently unparasitised red blood cell, depressed as well as ineffective erythropoiesis with dyserythropoietic changes and anaemia of chronic disease $[12,13]$.

Singh A et al., conducted a study on 200 cases, all of which showed abnormal scattergrams in cases of malaria, while abnormal WBC histogram peaks were noted in 96\% cases demonstrating a peak at the threshold of the histogram. Among all the haematological parameters, thrombocytopenia ( $92 \%$ cases) emerged as the strongest predictor of malaria $[5,9]$.

In the present study, $97.2 \%$ and $90.5 \%$ of abnormal histograms and abnormal scattergrams were seen respectively among the malaria positive cases.

The WBC-DIFF channel abnormalities were seen in 28 of 31 cases (sensitivity 90\%) among $P$. vivax patients. In the $P$. falciparum group, the WBC-DIFF channel abnormalities were seen in 4 of 8 cases (sensitivity 50\%). Of the 39 cases, 19 cases showed prominent bluecoded events in the area III in the WBC/BASO channel, as compared to the control group $(n=51)$ which showed such findings in only 3 of 40 cases [8].

A study on automated detection of malaria with haematology analyser sysmex xe-2100 shows that 52 out of 70 malaria positive cases showed abnormal scattergram in flowcytometric analyser. Good number of abnormal patterns in DIFF scattergram were observed in positive cases such as double neutrophil, double eosinophil, extended neutrophil with decreased space between eosinophil and neutrophil, grey zones, and the combination among the above. Extended neutrophil with decrease space between neutrophil and eosinophil was the commonest pattern followed by the grey zone [14].

Sharma $S$ et al., found that graying of both eosinophil and neutrophil groups (41.10\%) was the commonest abnormality followed by two eosinophil populations (16.20\%), overlapping of neutrophil and eosinophil groups (16.20\%), two neutrophil populations (14.70\%), graying of both lymphocyte and monocyte groups (8.50\%), graying of all leucocyte groups (2.30\%), and two lymphocyte populations (0.7\%). Among the $4.90 \%$ abnormalities in P. vivax, $22.20 \%$ abnormalities of $P$. falciparum were showing graying of lymphocyte and monocyte groups [5].

Mohapatra S et al., conducted a study on 40 cases and found that, five cases were reported negative by initial peripheral smear examination which had low parasite index, and these cases showed scattergram abnormalities, which were proved as positive on repeat peripheral smear [14].

Malaria can be predicted in patients who present with a combination of anaemia and thrombocytopenia. The next combination would be thrombocytopenia and monocytosis, though the latter is less sensitive and specific [15].

Gupta P et al., conducted a study on characterisation of platelet count and platelet indices in malaria patients and found that thrombocytopenia was significantly seen in $89.3 \%$ of malaria cases, both $P$. vivax and $P$. falciparum. Platelet count and PCT can be used as markers of disease severity [16].

Though malaria is a preventable and treatable disease, global burden of the cases still tend to be high, with one of the leading cause of death in children less than 5 years of age. Our study has basically 
focussed on the parameters which can help in suspecting malaria early and to make vigilant search for the parasite on peripheral smear to prevent further complications.

In present study, thrombocytopenia was seen in 94.5\%, normal MPV in $83.7 \%$, decreased PCT in 100\% and increased PDW in 98.6\% of malaria positive cases. Since these changes alone can be seen in many other conditions, none of them prove to be specific for the diagnosis, although these parameters along with abnormal scattergram and histogram play an important role in the early diagnosis of malaria, thus reducing the morbidity and mortality associated with malaria.

\section{LIMITATION}

Since the study was done for a short period of eight months, only 74 positive cases and their patterns on scattergram, histogram and platelet indices were studied. Hence to validate the study, a large population-based study is necessary.

\section{CONCLUSION}

Early diagnosis and treatment of malaria is necessary to prevent the complications associated with malaria. An abnormal scattergram, abnormal histogram along with thrombocytopenia, low PCT and increased PDW can predict presence of malaria. Though most of the laboratories are using cell counters, very few know and apply the specific patterns of malarial infection in the histogram, scattergram and platelet indices in the early diagnosis and hence early effective management.

\section{REFERENCES}

[1] Rathod DA, Patel V, Kaur AA, Patel VD, Patel DD. Diagnosis of acute malaria by laser based cell counter with comparison of conventional and recent techniques in Indian scenario. Indian J Pathol Microbiol. 2009;52:185-88.
[2] Maia M, Frank H. India's approach to eliminating malaria: A modeling perspective. J Biol Syst. 2010;18:867-91.

[3] Tangpukdee N, Duangdee C, Wilairatana P, Krudsood S. Malaria diagnosis: A brief review. Korean J Parasitol. 2009;47(2):93-102.

[4] James C, Sungano M, Philip T, Nirbhay K. A synopsis of current malaria diagnosis trends. Medical Journal of Zambia. 2009; 36: 95-101.

[5] Sharma S, Sethi N, Mukta P, Shivani K, Shivali S. Abnormal WBC scattergram: A clue to the diagnosis of malaria. Hematology. 2013;18(2):101-05.

[6] Shamim A, Gumashta R, Sadhana M, Sabiha M. Hematological changes in malaria: A comparative study. J Pharm Bio Sci. 2012;2(4):15-19.

[7] World Health Organization. New Perspectives: Malaria Diagnosis: Report of a Joint WHO/USAID Consultation, 25-27 October 1999. Geneva, Switzerland: World Health Organization; 2000.

[8] Monica Jain M, Gupta S, Jain J. Usefulness of automated cell counter in detection of malaria in a cancer set up-Our experience. Indian Journal of Pathology and Microbiology. 2012; 55 (2):467-73.

[9] Singh A, Narang V, Sood N, Garg B, Gupta VK. Malaria diagnosis using automated analysers: A boon for hematopathologists in endemic areas. J Clin Diagn Res. 2015;9(10):EC05-EC08

[10] Leal-Santos FA1, Silva SB, Crepaldi NP, Nery AF, Martin TO, Alves-Junior ER, et al. Altered platelet indices as potential markers of severe and complicated malaria caused by Plasmodium vivax: A cross-sectional descriptive study. Malar J. 2013;12:462.

11] Mubeen KH, Devadoss CW, Rangan RA, Gitanjali M, Prasanna S, Sunitha V. Automated hematology analyzers in diagnosis of plasmodium vivax malaria: an adjunct to conventional microscopy. Mediterr $J$ Hematol Infect Dis. 2014;6(1):e2014034.

[12] Clark IA, Chaudhri G. Tumour necrosis factor may contribute to the anemia of malaria by causing dyserythropoiesis and erythrophagoytosis. Brit J Haematol. 1988;70:99-103.

[13] Angus BJ, Chotivanich K, Silamut K, Ruangveerayuth R, Hardeman MR. Red blood cell deformability as a predictor of anemia in severe Falciparum malaria. Am J Trop Med Hyg. 1999;60:733-37.

[14] Mohapatra S, Samantaray J, Aruselvi S, Panda J, Munot K, Saxena R. Automated detection of malaria with hematology analyzer sysmex xe-2100. Indian Journal of Medical Sciences. 2011;65(1):26-31.

[15] Maina RN, Walsh D, Gaddy C, et al. Impact of infection on haematologica parameters in children living in Western Kenya. Malar J. 2010;9(3):4.

[16] Gupta P, Guddattu V and Kavitha Saravu K. Characterization of platelet count and platelet indices and their potential role to predict severity in malaria. Pathogens and Global Health. 2019;113(2):86-93.

\section{PARTICULARS OF CONTRIBUTORS:}

1. Assistant Professor, Department of Pathology, BMCRI, Bengaluru, Karnataka, India.

2. Consultant, Department of Pathology, Clumax Diagnostics, Bengaluru, Karnataka, India.

NAME, ADDRESS, E-MAIL ID OF THE CORRESPONDING AUTHOR:

Dr. G Prashanti,

\#776, $7^{\text {th }}$ Main, Narmada Nadhi road, Brindavan Nagar, Bengaluru-560029, Karnataka, India.

E-mail: shanthigogulas@gmail.com

Date of Submission: Jul 09, 2019

Date of Peer Review: Jul 24, 2019

Date of Acceptance: Aug 22, 2019

Date of Publishing: Oct 01, 2019 